\title{
Pathogenetic Significance of Myxomatous Degeneration in Fenestration-Related Massive Aortic Regurgitation
}

\author{
Kazuya Akiyama, MD; Jun Hirota, MD; Naohito Taniyasu, MD; Kazuma Maisawa, MD; \\ Yutaka Kobayashi, MD; Masatoshi Tsuda, MD
}

\begin{abstract}
Background Aortic valvular regurgitation has several mechanisms and the present study investigated its clinicopathological correlations with aortic valve fenestration.

Methods and Results Six male patients with massive regurgitation and enlarged fenestrations or ruptured fenestrated fibrous cords underwent aortic valve replacement. The clinicopathological features showed many similarities. Four cases had family histories of aortic regurgitation. All six patients showed moderate to severe myxomatous degeneration of the aortic valve and enlargement of aortic annulus. Four patients had 1-2 ruptured fibrous cords, located at the right coronary cusp. Echocardiographic examinations showed an abnormal fibrous cord attached to the prolapsing cusp in 3 cases with ruptured fenestrated valve and detailed examination by transesophageal echocardiography showed an intact fenestrated fibrous cord at the commissure in 1 case.

Conclusion Extensively fenestrated cusps with an enlarged aortic annulus because of myxomatous degeneration can cause chronic regurgitation or sudden deterioration after rupture of the fibrous cord. There is an increasing incidence of fenestration-related aortic regurgitation in the Japanese population. An important pathogenetic factor in male patients is myxomatous degeneration of the aortic cusp and annulus. (Circ J 2004; 68: 439-443)
\end{abstract}

Key Words: Aortic annular enlargement; Aortic valve regurgitation; Fenestration; Fibrous cord; Myxomatous degeneration

$\mathbf{F}$ enestration of the aortic valve is not an uncommon malformation, and appears as an oval hole immediately below the free edge of the cusp near its commissural attachment. Fenestration of the aortic cusp is not considered to have any pathological significance, and is rarely associated with aortic regurgitation, but we are operating on an increasing number of cases of massive aortic regurgitation in which large fenestrations play an important role in the pathogenesis of regurgitation. We present 6 cases in which the clinicopathological findings were analyzed.

\section{Methods}

\section{Patients}

During the past 9 years at Iwaki Kyoritsu General Hospital, more than 200 aortic valves have been replaced because of aortic regurgitation caused by idiopathic annular dilatation, infected endocarditis, rheumatic valvular disease or congenital bicuspid valve. Of these patients, 6 cases with large aortic valve fenestrations (Fig 1), which played an important role in producing massive aortic regurgitation, were evaluated from the clinical and pathological viewpoints. None of them showed systemic connective tissue disease or annuloaortic ectasia. The interval since onset was defined as the time span to surgical treatment from the appearance of new aortic regurgitant murmur, the

(Received October 1, 2003; revised manuscript received January 26, 2004; accepted February 9, 2004)

Department of Cardiovascular Surgery, Iwaki Kyoritsu General Hospital, Iwaki, Japan

Mailing address: Kazuya Akiyama, MD, Department of Cardiovascular Surgery, Iwaki Kyoritsu General Hospital, 16 Kusehara Uchigomimayamachi, Iwaki City, Fukushima 973-8555, Japan. E-mail: mick-aki@orion.ocn.ne.jp first experience of cardiac symptoms or echocardiographic detection of aortic regurgitation. Left ventricular hypertrophy was defined electrocardiographically by the QRS voltage criteria including $\mathrm{R}_{\mathrm{I}}+\mathrm{S}_{\text {III }} \geq 2.5 \mathrm{mV}, \mathrm{R}$ in $\mathrm{aV}$ $>2.0 \mathrm{mV}, \mathrm{S}$ in $\mathrm{V}_{1} \geq 2.4 \mathrm{mV}, \mathrm{R}$ in $\mathrm{V}_{5}$ or $\mathrm{V}_{6}>2.6 \mathrm{mV}$, and $\mathrm{R}$ in $\mathrm{V}_{5}$ or $\mathrm{V}_{6}+\mathrm{S}$ in $\mathrm{V}_{1}>3.5 \mathrm{mV}$. The aortic annular diameter (AAD), left ventricular diastolic dimension (LVDd), and left ventricular systolic dimension (LVDs) were measured by 2-dimensional echocardiography in the parasternal long-axis view. The severity of the aortic regurgitation was graded as trivial (I), mild (II), moderate (III) or severe (IV) using color flow mapping in the apical view of transthorac-

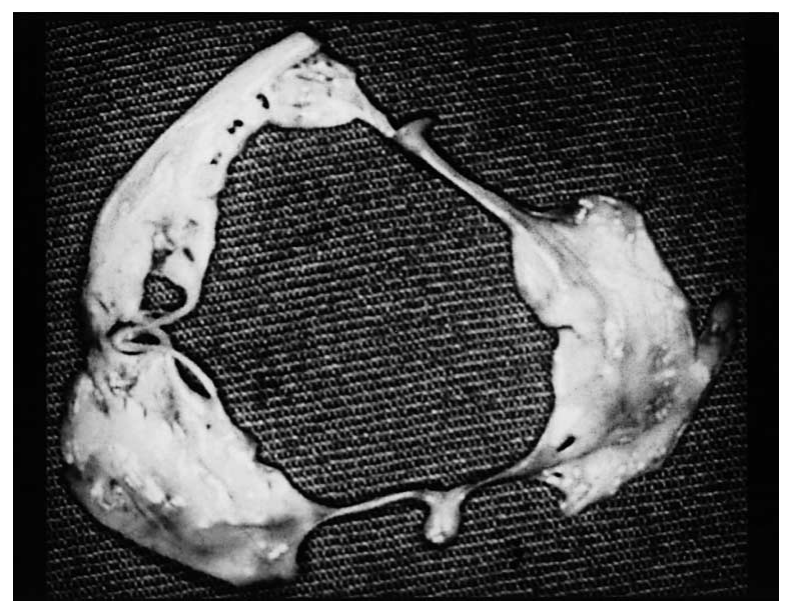

Fig 1. Patient 1 . The 3 aortic cusps (upper cusp=right coronary cusp; left lower cusp = left coronary cusp; right lower cusp=non-coronary cusp) have large fenestrations and intact fenestrated fibrous cords. 
Table 1 Clinical and Laboratory Findings of the Patients With Fenestrated Aortic Valves

\begin{tabular}{|c|c|c|c|c|c|c|}
\hline & \multicolumn{6}{|c|}{ Patient no. } \\
\hline & 1 & 2 & 3 & 4 & 5 & 6 \\
\hline Age (years) & 42 & 43 & 68 & 68 & 59 & 51 \\
\hline$H(\mathrm{~cm}) / W(\mathrm{~kg})$ & $155 / 53$ & $184 / 65$ & $166 / 59$ & $159 / 58$ & $166 / 68$ & $176 / 75$ \\
\hline $\mathrm{FH}$ & + & $+($ Marfan $)$ & + & + & - & - \\
\hline$H T$ & - & + & + & + & - & - \\
\hline NYHA & II & IV & III & III & III & III \\
\hline Interval & $6 y$ & $3 w$ & $3 y$ & $2 m$ & $2 m$ & $2 m$ \\
\hline $\mathrm{SAP}(\mathrm{mmHg})$ & $100 / 70$ & $182 / 84$ & $134 / 60$ & $170 / 50$ & $120 / 66$ & $130 / 82$ \\
\hline$E C G / L V H$ & + & + & + & + & + & + \\
\hline$U C G / A A D(\mathrm{~mm})$ & 36.8 & 30.0 & 32.4 & 31.0 & 28.6 & 30.2 \\
\hline LVDd/Ds (mm) & $61 / 42$ & $70 / 54$ & $67 / 47$ & $69 / 51$ & $67 / 49$ & $73 / 50$ \\
\hline Detection of FS & - & $+(T T E)$ & - & $+(T E E)$ & $+(T E E)$ & $+(T E E)$ \\
\hline$A R$ & $I V$ & IV & III & $I V$ & III & IV \\
\hline LVEDP $(\mathrm{mmHg})$ & 13 & $20 *$ & 32 & 29 & 20 & 23 \\
\hline$L V G / E D V I\left(\mathrm{ml} / \mathrm{m}^{2}\right)$ & 182 & $N E$ & 215 & 271 & 202 & 208 \\
\hline $\operatorname{ESVI}\left(\mathrm{ml} / \mathrm{m}^{2}\right)$ & 60 & $N E$ & 96 & 155 & 123 & 91 \\
\hline$E F(\%)$ & 67 & $N E$ & 55 & 43 & 47 & 61 \\
\hline
\end{tabular}

H, stature; W, body weight; FH, family history of aortic regurgitation; HT, history of systemic hypertension; NYHA, functional classification of New York Heart Association; Interval, interval from onset to aortic valve replacement; $y$, years; w, weeks; $m$, months; SAP, systemic arterial pressure; ECG, electrocardiogram; LVH, left ventricular hypertrophy; LVEDP, left ventricular enddiastolic pressure; UCG, ultrasonic echocardiographic findings; AAD, aortic annular diameter; Dd, left ventricular diastolic dimension; Sd, left ventricular systolic dimension; FS, fenestrated fibrous strand; AR, aortic regurgitation; TTE, transthoracic echocardiography; TEE, transesophageal echocardiography; LVG, left ventriculographic findings; EDVI, end-diastolic volume index; ESVI, end-systolic volume index; $E F$, ejection fraction; $N E$, not evaluated.

*Pulmonary capillary wedge pressure.

a

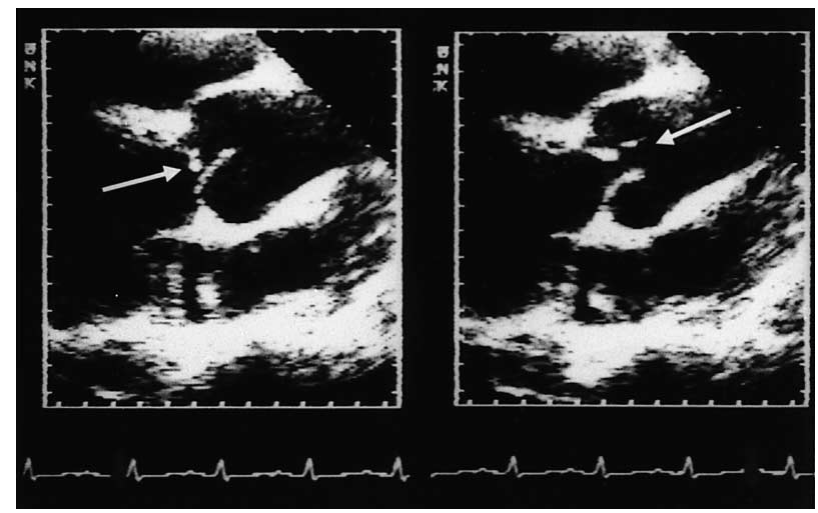

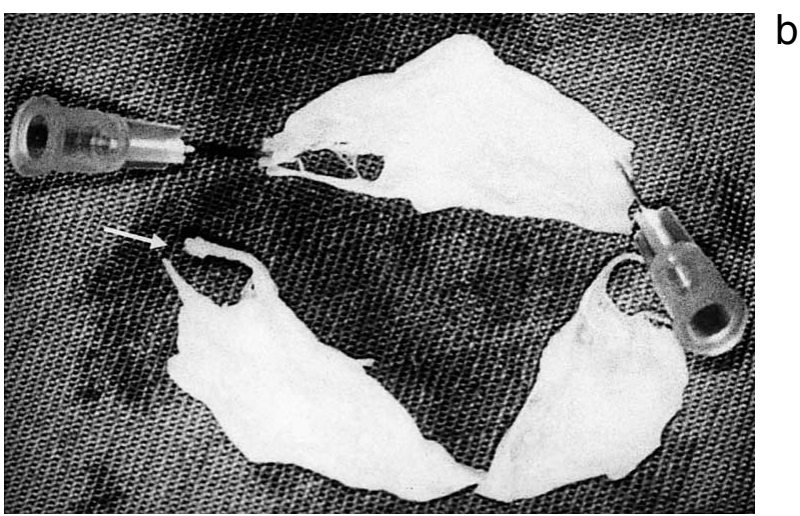

Fig 2. Patient 2. (a) Echocardiographic findings of the ruptured fenestrated aortic valve. The transthoracic echocardiogram shows an abnormal fibrous-strand echo of the right coronary cusp in systole (Right) and a prolapsed right coronary cusp (Left). (b) Excised aortic non-coronary cusp (Upper), right coronary cusp (Left lower), and left coronary cusp (Right lower). (Arrow) Torn fibrous cord.

ic echocardiography (TTE). Aortic valvular lesions were reevaluated by transesophageal echocardiography (TEE) intraoperatively. The left ventricular volume was calculated by the area-length method on single-plane views. Histological sections of formalin-fixed aortic cusp samples were stained with the hematoxylin-eosin and the elastica Masson stain for elastin and collagen, and alcian blue/PAS stain for acid mucopolysaccharides. The severity of myxomatous degeneration was graded by the pathologist from mild (I), moderate (II), to severe (III) according to the presence of increased amounts of acid mucopolysaccharides: mild, $<25 \%$ of the zona spongiosa; moderate, $25-50 \%$ of the zona spongiosa; severe, $>50 \%$ of the zona spongiosa.

\section{Results}

Clinical and Laboratory Findings (Table 1)

At the time of aortic valve replacement, the age of the 6 male patients ranged from 42 to 68 years (mean, 53.3 years). Preoperatively, 1 patient was in functional class II of the New York Heart Association classification (NYHA), 4 patients were in class III, and 1 was in class IV. The one patient in class II underwent aortic valve replacement because of increasing massive aortic regurgitation with an enlarged left ventricular cavity. None of the 6 patients had any history of infective endocarditis or chest trauma, nor did they have a positive serological test for syphilis. Four patients had family histories of aortic valvular disease and one of them had a family history of Marfan syndrome. Three patients had a past history of systemic hypertension requiring oral hypotensive drugs and 1 patient had hypotension without treatment. The other 2 patients were normotensive. The patient with the family history of Marfan syndrome did not show any other specific manifestation for this syndrome except for tall stature. Four patients had sudden onset of cardiac symptoms, including nocturnal 
a

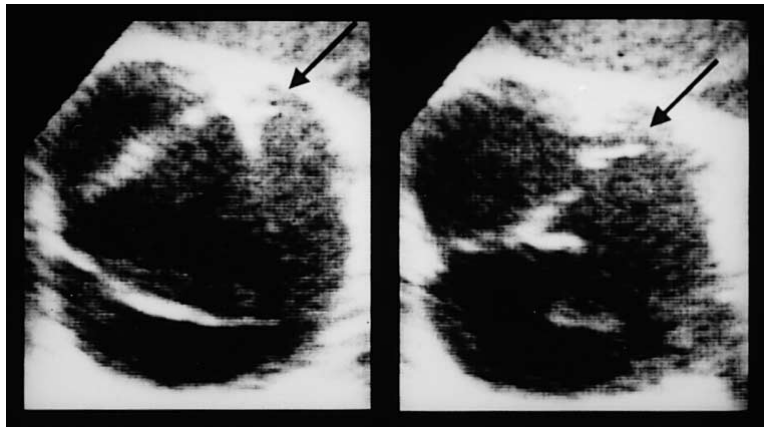

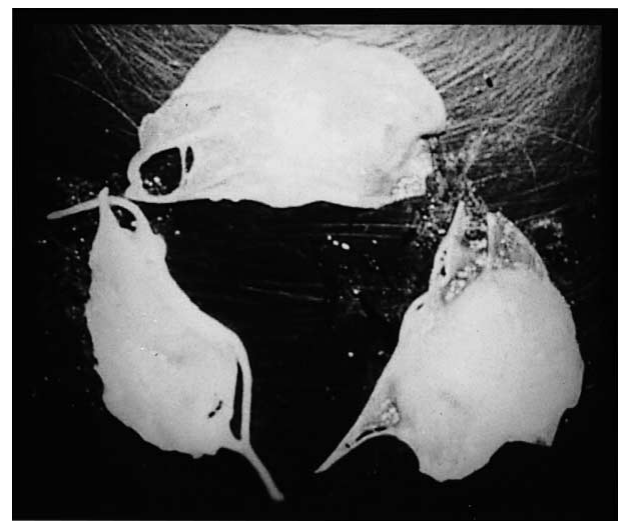

Fig 3. Patient 4. (a) Transesophageal echocardiogram shows an abnormal fibrous band in the commissure between the left and non-coronary cusps (arrow). (Right) Diastolic phase, (Left) systolic phase. In diastole, each cusp with extra length at the free margin is well coapted without the fenestrated site. (b) The excised three aortic cusps: right coronary cusp (Upper), left coronary cusp (Left lower), non-coronary cusp (Right lower). The fenestrated fibrous cords are intact.

Table 2 Operative and Pathological Findings of the Patients With Fenestrated Aortic Valves

\begin{tabular}{lcccccc}
\hline \hline & \multicolumn{7}{c}{ Patient no. } \\
\cline { 2 - 7 } & 1 & 2 & 3 & 4 & 5 & 6 \\
\hline Site of $F$ & $R, L, N$ & $R, L, N$ & $R, L, N$ & $R, L, N$ & $R, L, N$ & $R, L$ \\
Ruptured cusp & - & $R$ & $R, N$ & - & $R$ & $R$ \\
Size of AA $($ mm $)$ & $32<$ & 28 & $32<$ & 30 & 27 & 30 \\
Size/PV & $27 / S-J$ & $27 / S-J$ & $25 / S-J$ & $25 / C-M$ & $25 / S-J$ & $25 / S-J$ \\
MXD of $A$ Ao & $N E$ & + & + & $N E$ & + & $N E$ \\
Grade of $M X D$ & ++ & +++ & +++ & ++ & +++ & +++
\end{tabular}

$F$, fenestration; $R$, right coronary cusp; L, left coronary cusp; $N$, non-coronary cusp; $A A$, aortic annulus measured by the seizures; $P V$, prosthetic valve; S-J, St Jude Medical Mechanical Valve; C-M, Carbomedics Mechanical Valve; Ao, aorta; NE, not evaluated; $M X D$, myxomatous degeneration.

++ moderate; +++ , severe.

dyspnea, dyspnea on excursion or awareness of heart murmur, and these gradually progressed in 1 patient (patient 3 ). One patient (patient 1) was free of cardiac symptoms despite increasing aortic regurgitation and cardiac enlargement. Two patients underwent surgery more than 3 years after the detection of regurgitation and the other 4 patients had surgery within 2 months of the onset of cardiac symptoms.

In all patients, the electrocardiographic findings were left ventricular hypertrophy and the ultrasound echocardiographic findings showed moderate to severe aortic regurgitation with an enlarged aortic annular diameter, ranging from 28.6 to $36.2 \mathrm{~mm}$ (mean, $31.8 \mathrm{~mm}$ ); however, ascending aortic aneurysm was not detected in any of them. In 1 case, preoperative TTE showed suspicious findings of a ruptured fibrous cord (patient 2) (Fig 2a,b), and intraoperative TEE showed a non-ruptured fibrous cord at the commissure in patient 4 (Fig 3a,b) and ruptured fibrous cord in 2 cases (patients 5 and 6). Ruptured fibrous cords flailing with the aortic cusp could be detected in the diastolic phase in patient 6 and the systolic phase in 2 patients (patients 2 and 5). The LVDd measured at the mid-portion of the left ventricle ranged from 73 to $61 \mathrm{~mm}$, with a mean value of $67.8 \mathrm{~mm}$. Data obtained during cardiac catheterization of 5 patients showed elevated LV end-diastolic pressure with a mean value of $23.4 \mathrm{mmHg}$, ranging from 13 to $32 \mathrm{mmHg}$, but did not show a significant pressure gradient of the aortic valve. The LV end-diastolic volume index showed a mean value of $215.6 \mathrm{ml} / \mathrm{m}^{2}$, ranging from 182 to $271 \mathrm{ml} / \mathrm{m}^{2}$ and the mean ejection fraction was $54.6 \%$, ranging from 43 to $67 \%$.

\section{Operative and Pathological Findings (Table 2)}

Surgical treatment, under cardiopulmonary bypass and mild hypothermia, was elective in 5 patients and emergency in one. In all cases the aortic valves were composed of 3 cusps. None of them showed annuloaortic ectasia or infective endocarditis. Fenestrations were located within every cusp at all 3 commissures in the 5 patients, and the other case had fenestrations within only 2 cusps. However, the right coronary cusps were fenestrated in all 6 cases. One fenestrated fibrous cord was torn in 3 cases and 2 fibrous cords of 2 different cusps located at the same commissure were torn in 1 case. The fenestrated fibrous cords were intact in 2 patients. The sites of the ruptured fenestrated fibrous cords were on the right coronary cusp in 4 cases (Fig 4). One of them had the other ruptured fibrous cord on the non-coronary cusp. The aortic valves were replaced with $25 \mathrm{~mm}$ St Jude Medical mechanical valve prostheses in 3 patients, a $25 \mathrm{~mm}$ Carbomedics mechanical valve in 1 , and a $27 \mathrm{~mm}$ St Jude Medical mechanical valve in the other 2 patients. The implanted valves, averaging $25.7 \mathrm{~mm}$ in size, were considerably larger than is usually required for Japanese patients.

The histopathological findings of the excised aortic valves showed moderate to severe myxomatous degeneration in all 6 cases (Fig 5) and that of aortic walls examined in 3 patients, including the patient with a family history of Marfan syndrome (patient 2), also showed myxomatous degeneration. The end of the ruptured fenestrated fibrous cord showed severe myxomatous degeneration (Fig 6).

The postoperative course was uneventful in each patient 

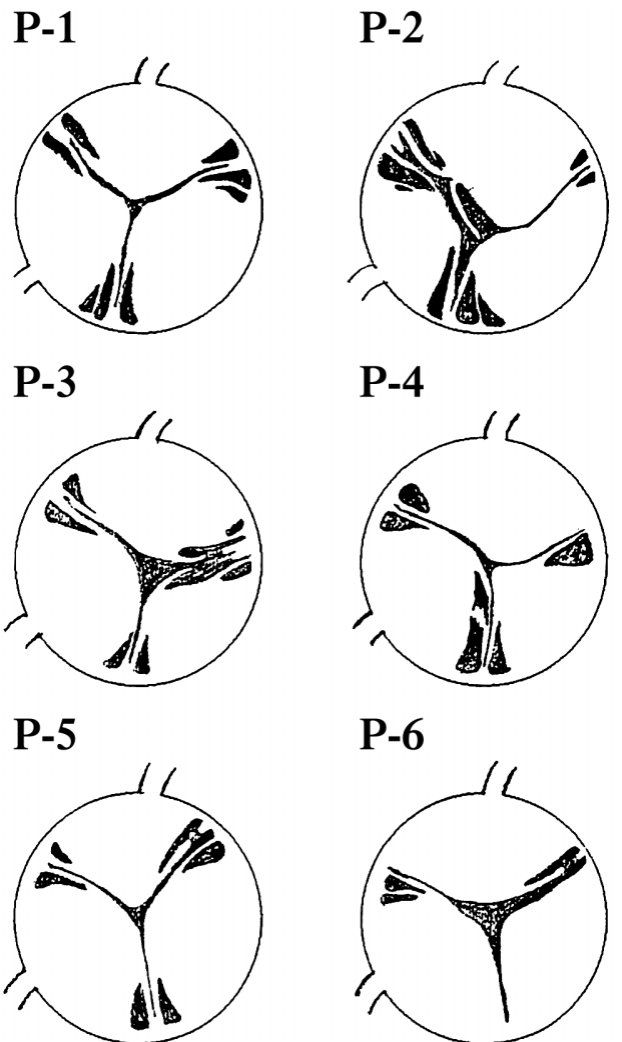

Fig 4. Schemata of each of the 6 fenestrated aortic valves. P-, patient.

and they remain well as outpatients being treated with warfarin.

\section{Discussion}

The small fenestrations of the aortic valve form during early embryonic phase as a developmental anomaly, similar to development of the chorda in the atrioventricular valve, resulting from undermining of the trabeculated ventricular wall! The incidence and severity of fenestration is similar for black and white races, but is considerably higher in males? An anomalous fibrous cord, located between the aortic cusp and the aortic wall, is able to produce massive and significant aortic regurgitation in both the bicuspid and tricuspid aortic valves, and most such cases have been reported from institutes in Japan? Apart from the present 6 cases with large fenestrations in the tricuspid aortic valve, during the past 9 years we have operated on 4 cases of a large fenestrated raphe in the bicuspid aortic valve4 It appears that there is a higher incidence in Japan of aortic valve regurgitation associated with anomalous fibrous cords than in the North America and European populations 3,5 The aortic valve is affected by fenestration slightly more frequently than the pulmonary valve, but each valve is involved in more than half the subjects. With widening of the annular ring, there is a definite increase in the severity and frequency of fenestration in both of the semilunar valves? Fenestrations, which are commonly located in the commissures, are not usually thought to be of functional significance, although they can occur to a varying degree during aging6 Although large aortic valve fenestrations can produce chronic regurgitation? rupture of the fenestrated fibrous cord, either spontaneously ${ }^{8}$ or

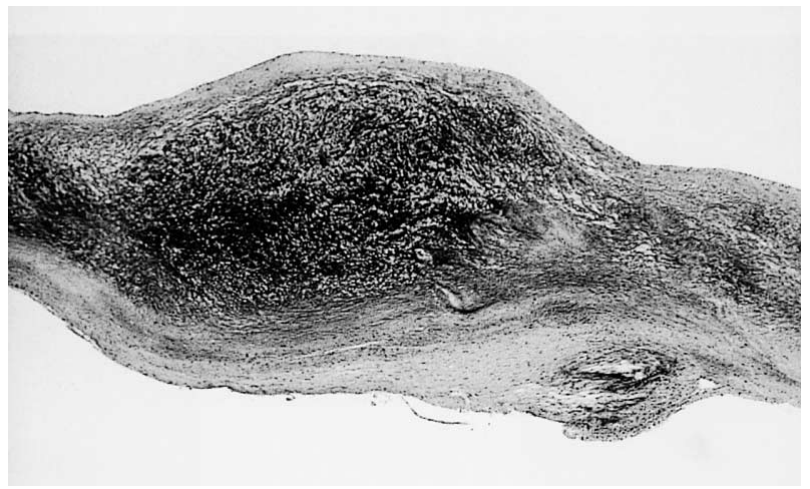

Fig 5. Pathological findings of the aortic cusp from patient 3 . The zona spongiosa is entirely affected by the deposition of acid mucopolysaccharides and shows severe myxomatous degeneration (alcian blue/PAS stain, $\times 50$ ).

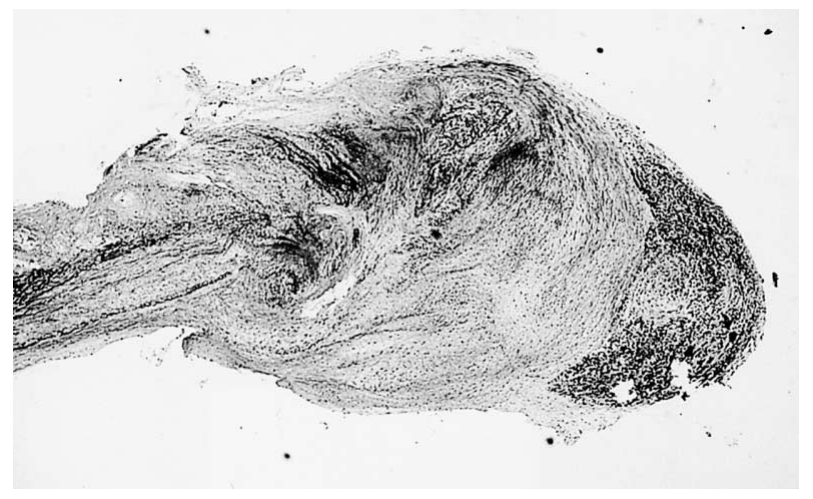

Fig 6. Pathological findings of the ruptured fenestrated fibrous cord from patient 6 . There is myxomatous degeneration of the entire body of the cord with bleeding at the ruptured end (elastica Masson, $\times 50$ ).

because of infection, can lead to massive regurgitation resulting in acute left heart failure.

Myxomatous degeneration of the aortic valve has the macroscopic characteristics of thin and translucent regions in each leaflet. Microscopically, the changes are accompanied by disruption of the fibrosa resulting from fragmentation of collagen fibers and accumulation of acid mucopolysaccharides in the spongiosa? Krovetz demonstrated that aortic valve size increases almost linearly with age after approximately 20 years old and that the rate of aortic dilatation in males is faster than in females 10 The aortic annular diameters in the present subjects were larger than in cases treated with valve replacement because of pure regurgitation, 10 although the latter valves are much larger than that of normal subjects! $!^{2}$ In normally functioning aortic valves, the sum of the area of the aortic valve cusps is said to increase with age $!^{3}$ The enlarged aortic annulus, which can also be affected by myxomatous degeneration, would facilitate enlargement of the fenestrations rather than enlargement of the cuspal area ${ }^{14,15}$ We think that the chronic aortic regurgitation in the present cases resulted not only from the large fenestrations at the commissures, but also from incomplete coaptation of the stretched leaflets caused by annular dilatation.

Some authors suggest that myxomatous degeneration of the aortic valve might be secondary to long-standing systemic hypertention ${ }^{10}$ but others state that the hypertension 
is not common in patients with myxomatous degeneration of the aortic valve ${ }^{6}$ Marcus et al thought systemic hypertension was an important factor in the spontaneous rupture of the fenestrated aortic valve ${ }^{16}$ because fenestration of the pulmonary valve, which occurs with almost the same frequency as that of the aortic valve, seldom progresses to rupture, because of the lower valve stress. However, our data showed that systemic hypertension is not always an essential factor in the development of myxomatous degeneration and rupture of the fenestrated fibrous cord in cases with a large fenestration. In addition, half the subjects, of which 2 had rupture of a fenestrated fibrous cord and 1 had intact fenestrations, had no history of systemic hypertension. The pathological finding was severe myxomatous degeneration of the aortic valve in the cases with a ruptured fibrous cord, so we consider that degeneration of the collagen within the central core of the fenestrated fibrous cord of the aortic valve was primarily responsible for enlargement of the fenestration and rupture of the fenestrated fibrous cord, similar to the chordal elongation and torn chorda of the mitral valve observed with severe myxomatous degeneration.

Our clinical data suggest that fenestration-related massive regurgitation will be only be detected in male patients and as 4 of the 6 cases had a family history of aortic regurgitation, there may be a hereditary origin in massive regurgitation caused by fenestration. Although the fenestrations were located in almost every cusp and at the commissure, the site of the ruptured fenestrated fibrous cord was always on the right coronary cusp. The reason for this may be that the right coronary cusp, which is poorly supported by the cardiac fibrous skeleton holding the valves together, is subjected to the annular elongation of the right coronary cusp and also can be exposed to more turbulent diastolic flow than the other aortic cusps. The average width of the right coronary cusp is larger than that of the other 2 cusps ${ }^{18}$ So fenestrations and the fenestrated fibrous cord of the right coronary cusp would be stretched and ruptured more than those of the other 2 coronary cusps.

With the decreasing incidence of aortic valve disease caused by rheumatic fever, aortic regurgitation results from other, uncommon causes. In older subjects, the number and area affected by fenestrations of the aortic valve tends to increase and we believe that in the near future, aortic valve fenestrations will play an important role in the development of chronic aortic regurgitation or its sudden worsening in adult male patients. Therefore, physicians must be aware of the fenestrated aortic valve in a male patient with chronic aortic regurgitation or sudden deterioration of regurgitation, if there is not any other obvious cause. The essential anatomic features of aortic valve disease can be clearly defined by high-resolution imaging by 2-dimensional echocardiography, and close examination using TTE and TEE is very useful for detecting a fenestrated aortic valve. Echocardiographic examination accurately provides quantitative and qualitative assessment of aortic regurgitation ${ }^{19,20}$ and the findings that are suggestive of regurgitation caused by a fenestrated aortic valve are an enlarged aortic annulus with a diameter greater than $30 \mathrm{~mm}$, a prolapsing right coronary cusp with/without eccentric regurgitation, and an abnormal fibrous band attached to the prolapsing cusp or fibrous band between the aortic cusp and the aortic wall at the commissure. Needless to say, aortic valve replacement is essential unless the central fenestration of the cusp is caused by infective endocarditis.

\section{Acknowledgment}

We are grateful to Dr Shigeyuki Asano for his valuable comments on the pathological findings of the aortic valves.

\section{References}

1. Foxe AN. Fenestrations of the semiluna valves. Am J Pathol 1929; 5: $179-182$.

2. Friedman B, Hathaway BM. Fenestration of the semilunar cusps, and "functional" aortic and pulmonic valve insufficiency. Am J Med 1958; 24: 549-558.

3. Yavuz S, Türk T, Celkan A, Koca V, Ata Y, Ozdemir IA. Congenital aortic insufficiency due to aortic cusp stretching: 'Kite anomaly'. $J$ Heart Valve Dis 1999; 8: 284-286.

4. Akiyama K, Hirota J, Taniyasu N, Maisawa K, Sasaki S, Takazawa A, et al. Clinical implication of fenestrated raphe in congenital bicuspid aortic valve with severe regurgitation. Iwaki Kyoritsu Iho 2002; 23: $11-16$.

5. Nakajima M, Tsuchiya K, Naito $Y$, Hibino N, Inoue H. Aortic regurgitation caused by rupture of a well-balanced fibrous strand suspending a degenerative tricuspid aortic valve. J Thorac Cardiovasc Surg 2002; 124: 843-844.

6. Lester WM. Age-related cardiovascular changes. In: Silber MD, Gotlieb AI, Schoen FJ, editors. Cardiovascular pathology, 3rd edn. Philadelphia: Churchill Livingstone; 2001; 54-67.

7. Kaplan J, Farb A, Carliner NH, Virmani R. Large aortic valve fenestrations producing chronic aortic regurgitation. Am Heart J 1991; 122: $1475-1477$.

8. Morán SV, Casanegra P, Maturana G, Dubernet J. Spontaneous rupture of a fenestrated aortic valve. Surgical treatment. J Thorac Cardiovasc Surg 1977; 73: 716-718.

9. Westlake G. Ruptured aortic cords. Aust NZ J Surg 1973; 43: 127 129.

10. Allen WM, Matloff JM, Fishbein MC. Myxoid degeneration of the aortic valve and isolated severe aortic regurgitation. Am J Cardiol 1985; 55: 439-444.

11. Westaby S, Karp RB, Blackstone EH, Bishop SP. Adult human valve dimensions and their surgical significance. Am J Cardiol 1984; 53: $552-556$.

12. Davies MJ. Pathology of cardiac valves. London: Butterworths; 1980; 49.

13. Silver MA, Roberts WC. Detailed anatomy of the normally functioning aortic valve in hearts of normal and increased weight. $A m J$ Cardiol 1985; 55: 454-461.

14. Flinger CL, Reichenbach DD. Pathology and etiology of heart disease. In: Otto CM, editor. Valvular heart disease. Philadelphia: WB Saunders; $1999 ; 13-42$.

15. Krovetz LJ. Age-related changes in size of the aortic valve annulus in man. Am Heart J 1975; 90: 569-574.

16. Tonnemacher D, Reid C, Kawanishi D, Cumming T, Chandrasoma P, McKay CR, et al. Frequency of myxomatous degeneration of the aortic valve as a cause of isolated aortic regurgitation severe enough to warrant aortic valve replacement. Am J Cardiol 1987; 60: $1194-$ 1196.

17. Marcus FI, Ronan J, Misanik LF, Ewy GA. Aortic insufficiency secondary to spontaneous rupture of a fenestrated leaflet. Am Heart J 1963; 66: 675-678.

18. Vollebergh FEMG, Becker AE. Minor congenital variations of cusp size in tricuspid aortic valves. Possible link with isolated aortic stenosis. Br Heart J 1977; 39: 1006-1011.

19. Nozaki S, Mizushige K, Tanimoto T, Obayashi N, Matsuo H. New index for grading the severity of aortic regurgitation based on the cross-sectional area of vena contracta measured by color Doppler flow mapping. Circ J 2003; 67: 243-247.

20. Tomita H, Yamada O, Kurosaki K, Yagihara T, Echigo S. Eccentric aortic regurgitation in patients with right coronary cusp prolapse complicating a ventricular septal defect. Circ J 2003; 67: 672-675. 